\title{
Gauge-Origin Independent Density-Functional Theory Calculations of Vibrational Raman Optical Activity
}

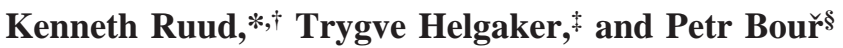 \\ Department of Chemistry, University of Troms $\phi$, N-9037 Troms $\phi$, Norway, Department of Chemistry, \\ University of Oslo, P.O. Box 1033 Blindern, N-0315 Oslo, Norway, and Institute of Organic Chemistry and \\ Biochemistry, Academy of Sciences of the Czech Republic, Flemingovo nam 2, 16610, Praha 6, Czech Republic
}

Received: April 29, 2002; In Final Form: June 14, 2002

\begin{abstract}
We present density-functional theory calculations of vibrational Raman optical activity. Gauge-origin independence of the results is ensured by using London atomic orbitals, and the frequency dependence is included by using analytical response theory. The Raman optical activity circular intensity differences are obtained by combining analytical calculations of linear response functions with numerical differentiation with respect to nuclear distortions. The results are compared with experiment, previous Hartree-Fock calculations, and a more recent approximate sum-over-states approach.
\end{abstract}

\section{Introduction}

The applicability of the Raman technique in solution combined with the stereochemical information provided by the sign of the circular vibrational Raman scattering intensity difference gives vibrational Raman optical activity (VROA) a great potential for structural studies of biomolecules. ${ }^{1-3}$ Nevertheless, since its discovery in the 1970s, VROA has not attracted much interest, mainly due to the lack of a commercially available spectrometer. Indeed, the first announcement of a commercially available VROA spectrometer was made only last year. ${ }^{4}$

Although the vibrational frequencies observed in a VROA spectrum can be assigned in much the same way as for an ordinary Raman spectrum, the sign of the circular intensity difference (CID) is difficult to predict a priori because of the lack of empirical rules for correlating the sign of the intensity to the absolute stereochemistry of the molecule. It can be expected that empirical rules for the CID sign in relation to stereochemistry will receive more attention as VROA becomes more widespread; currently, the use of theoretical methods for determining the sign of the CIDs in relation to the absolute stereochemistry of the molecule is needed for an unambiguous assignment. ${ }^{5}$

For several reasons, the theoretical calculation of VROA CIDs is a challenging task. First, because the property vanishes for a static electromagnetic field, either linear response methods 6,7 or the static-limit approximation introduced by $\mathrm{Amos}^{8}$ must be used. Second, the tensors contributing to the VROA intensities are third-order properties, requiring so far the use of an expensive numerical differentiation. ${ }^{7,9}$ Third, one of the contributing tensors involves the interaction with the magnetic component of the electromagnetic field, making approximate theoretical calculations gauge-origin dependent, this problem can be solved using London atomic orbitals. ${ }^{7,10-12}$ Fourth, it is well-known that Hartree-Fock harmonic force fields are of

\footnotetext{
$\dagger$ University of Tromsø.

$\doteqdot$ University of Oslo.

$\S$ Academy of Sciences of the Czech Republic.
}

rather poor quality, ${ }^{13,14}$ which might affect the intensities because the tensors are transformed to the normal coordinate basis. ${ }^{7,15}$

Only a few implementations of the full VROA CIDs have been presented, ${ }^{7,9}$ and the literature on calculations of VROA CIDs is still rather limited-see ref 5 for a review. Recently, Bouř and co-workers introduced an approximate sum-over-states (SOS) scheme that significantly reduces the time required for calculating VROA intensities. ${ }^{16,17}$ These schemes are sometimes combined with an approximate evaluation of some of the generalized polarizabilities involved in the VROA CID, known as the polar model, ${ }^{18}$ which we will denote as SOS+Polar. Their scheme has also used more accurate density-functional theory (DFT) force fields. ${ }^{17}$ In the SOS approach, the summation over states is truncated, thus introducing errors in the intensities, although the CID signs probably remain correct. However, in evaluating the performance of this approximate method, the CIDs obtained at the DFT level were compared with intensities calculated with a mixed numerical-analytical scheme at the Hartree-Fock level. This is unfortunate, as it becomes difficult to disentangle the errors arising from the SOS truncation from the errors inherent in the Hartree-Fock approximation.

In this paper, we present a mixed numerical-analytical scheme for calculating VROA intensities consistently by DFT. Gaugeorigin independence is ensured by the use of the London atomic orbitals ${ }^{10}$ and the dependence on the frequency is accounted for by linear response theory. ${ }^{6,7}$ The derivatives of the different generalized polarizabilities with respect to nuclear distortions are obtained by numerical differentiation of the analytically calculated polarizabilities. This work therefore combines our previous Hartree-Fock implementation of $\mathrm{VROA}^{7}$ with our recent implementation of gauge-origin independent optical rotation at the DFT level. ${ }^{19}$

The paper is organized as follows. In section 2, we give a brief summary of the theory for VROA calculations. In section 3 , we provide some technical details about the calculations presented in this paper. Section 4 summarizes our results and compares these to experimental observations and earlier theoretical works. Finally, we give some concluding remarks in section 5 . 


\section{Theory}

The theoretical foundation of VROA was developed by Barron and Buckingham, ${ }^{20}$ following the theoretical prediction of the scattering mechanism by Atkins and Barron. ${ }^{21}$ In particular, it was demonstrated that the VROA intensity is determined by the geometry dependence of three generalized polarizabilities-namely, the ordinary electric dipole-electric dipole polarizability

$$
\alpha_{\alpha \beta}=2 \sum_{n \neq 0} \omega_{n 0} \frac{\operatorname{Re}\left[\left\langle 0\left|\mu_{\alpha}\right| n\right\rangle\left\langle n\left|\mu_{\beta}\right| 0\right\rangle\right]}{\omega_{n 0}{ }^{2}-\omega^{2}}
$$

the mixed electric dipole-magnetic dipole polarizability

$$
G_{\alpha \beta}^{\prime}=-2 \sum_{n \neq 0} \omega \frac{\operatorname{Im}\left[\left\langle 0\left|\mu_{\alpha}\right| n\right\rangle\left\langle n\left|m_{\beta}\right| 0\right\rangle\right]}{\omega_{n 0}{ }^{2}-\omega^{2}}
$$

and the mixed electric dipole-electric quadrupole polarizability

$$
A_{\alpha \beta \gamma}=2 \sum_{n \neq 0} \omega_{n 0} \frac{\operatorname{Re}\left[\left\langle 0\left|\mu_{\alpha}\right| n\right\rangle\left\langle n\left|\Theta_{\beta \gamma}\right| 0\right\rangle\right]}{\omega_{n 0}{ }^{2}-\omega^{2}}
$$

Here $\omega_{n 0}$ is the excitation energy from the ground state $|0\rangle$ to the excited state $|n\rangle$ and $\omega$ is the frequency of the incident light. The summations are over all the (exact) electronically excited states of the molecule. In these equations, we have also introduced the electric dipole operator (atomic units used throughout)

$$
\mu_{\alpha}=-\sum_{i} r_{i \alpha}
$$

the magnetic dipole operator

$$
m_{\alpha}=-\frac{1}{2} \sum_{i} l_{i \alpha}
$$

and the electric quadrupole operator

$$
\Theta_{\alpha \beta}=-\frac{1}{2} \sum_{i}\left(3 r_{i \alpha} r_{i \beta}-r_{i}^{2} \delta_{\alpha \beta}\right)
$$

The summations are over all electrons, and $r_{\mathrm{i}}$ and $l_{\mathrm{i}}$ are the position and angular momentum of electron $i$, respectively.

Of particular interest for the calculation of VROA CIDs is the mixed electric dipole-magnetic dipole polarizability in eq 2. The magnetic dipole moment depends on the choice of gauge origin, leading to an unphysical dependence on this origin in approximate calculations. However, we have previously demonstrated that, for variational wave functions such as HartreeFock and multiconfigurational self-consistent field (MCSCF) wave functions, ${ }^{7}$ the exact origin dependence is recovered even for finite basis sets, provided London atomic orbitals are used. ${ }^{10,22}$ Note that, unlike its trace, the individual components of the electric dipole-magnetic dipole polarizability tensor are not gauge-origin independent, even for the exact wave function.

The generalized polarizability eq 2 appears also in calculations of optical rotation. ${ }^{23}$ Stephens and co-workers have demonstrated how the gauge-origin independent DFT calculation of optical rotation is achieved by the use of London atomic orbitals ${ }^{7,10}$ combined with time-dependent DFT. We recently implemented ${ }^{19}$ this formalism in the Dalton program system. ${ }^{24}$ To use this implementation of London-orbital DFT optical rotation ${ }^{19}$ for the calculation of VROA CIDs, we note that the generalized polarizabilities in eqs $1-3$ can be formulated as linear response functions ${ }^{6}$

$$
\begin{gathered}
\alpha_{\alpha \beta}=-\left\langle\left\langle\mu_{\alpha} ; \mu_{\beta}\right\rangle\right\rangle \\
G_{\alpha \beta}^{\prime}=-\mathrm{i}\left\langle\left\langle\mu_{\alpha} ; m_{\beta}\right\rangle\right\rangle_{\omega} \\
A_{\alpha \beta \gamma}=-\left\langle\left\langle\mu_{\alpha} ; \Theta_{\beta \gamma}\right\rangle\right\rangle_{\omega}
\end{gathered}
$$

Of particular interest here is the CID introduced by Barron and Buckingham $^{20}$

$$
\Delta_{\alpha}=\frac{I_{\alpha}^{\mathrm{R}}-I_{\alpha}^{\mathrm{L}}}{I_{\alpha}^{\mathrm{R}}+I_{\alpha}^{\mathrm{L}}}
$$

where $I_{\alpha}^{\mathrm{R}}$ and $I_{\alpha}^{\mathrm{L}}$ are the scattered intensities with $\alpha$ polarization for right and left circularly polarized light, respectively. More specifically, the CIDs for right-angle and backscattering are given as

$$
\begin{aligned}
\Delta_{z}\left(90^{\circ}\right) & =\frac{6 \beta\left(G^{\prime}\right)^{2}-2 \beta(A)^{2}}{3 c \beta(\alpha)^{2}} \\
\Delta_{z}\left(180^{\circ}\right) & =\frac{24 \beta\left(G^{\prime}\right)^{2}+8 \beta(A)^{2}}{3 c \beta(\alpha)^{2}}
\end{aligned}
$$

where we have expressed the CIDs in terms of the anisotropic invariants

$$
\begin{gathered}
\beta(\alpha)^{2}=\frac{1}{2}\left(3 \alpha_{\alpha \beta} \alpha_{\alpha \beta}-\alpha_{\alpha \alpha} \alpha_{\beta \beta}\right) \\
\beta\left(G^{\prime}\right)^{2}=\frac{1}{2}\left(3 \alpha_{\alpha \beta} G_{\alpha \beta}^{\prime}-\alpha_{\alpha \alpha} G_{\beta \beta}^{\prime}\right) \\
\beta(A)^{2}=\frac{1}{2} \omega \alpha_{\alpha \beta} \epsilon_{\alpha \gamma \delta} A_{\gamma \delta \beta}
\end{gathered}
$$

using implicit summation over repeated indices, $\epsilon_{\alpha \beta \gamma}$ is the unit third-rank antisymmetric tensor, and $c$ is the speed of light.

For the vibrational Raman scattering process, we do not need the generalized polarizabilities eqs $1-3$ but rather the corresponding vibrational transition moments. We do not repeat the derivations of ref 25 but note that, by carrying out a perturbation expansion of the generalized polarizability tensors and the vibrational wave function, and by invoking the Placzek approximation, ${ }^{26}$ we can approximate the vibrational transition moments as

$$
\begin{gathered}
\left\langle v_{0}\left|\alpha_{\alpha \beta}\right| v_{1 \mathrm{p}}\right\rangle\left\langle v_{1 \mathrm{p}}\left|\alpha_{\alpha \beta}\right| v_{0}\right\rangle=\left.\left.\frac{1}{2 \omega_{\mathrm{p}}}\left(\frac{\partial \alpha_{\alpha \beta}}{\partial Q_{\mathrm{p}}}\right)\right|_{r_{\mathrm{e}}}\left(\frac{\partial \alpha_{\alpha \beta}}{\partial Q_{\mathrm{p}}}\right)\right|_{r_{\mathrm{e}}} \\
\left\langle v_{0}\left|\alpha_{\alpha \beta}\right| v_{1 \mathrm{p}}\right\rangle\left\langle v_{1 \mathrm{p}}\left|G_{\alpha \beta}^{\prime}\right| v_{0}\right\rangle=\left.\left.\frac{1}{2 \omega_{\mathrm{p}}}\left(\frac{\partial \alpha_{\alpha \beta}}{\partial Q_{\mathrm{p}}}\right)\right|_{\mathrm{r}_{\mathrm{e}}}\left(\frac{\partial G^{\prime} \alpha_{\alpha \beta}}{\partial Q_{\mathrm{p}}}\right)\right|_{r_{\mathrm{e}}} \\
\left\langle v_{0}\left|\alpha_{\alpha \beta}\right| v_{1 \mathrm{p}}\right\rangle\left\langle v_{1 \mathrm{p}}\left|\epsilon_{\alpha \gamma \delta} A_{\gamma \delta \beta}\right| v_{0}\right\rangle=\left.\left.\frac{1}{2 \omega_{\mathrm{p}}}\left(\frac{\partial \alpha_{\alpha \beta}}{\partial Q_{\mathrm{p}}}\right)\right|_{r_{\mathrm{e}}} \epsilon_{\alpha \gamma \delta}\left(\frac{\partial A_{\gamma \delta \beta}}{\partial Q_{\mathrm{p}}}\right)\right|_{r_{\mathrm{e}}}
\end{gathered}
$$

In these equations, $v_{0}$ and $v_{1 \mathrm{p}}$ denote the vibrational ground state and the first vibrationally excited state for vibrational mode $\mathrm{p}$, 
TABLE 1: Calculated ROA Intensities for $\boldsymbol{R}$-Methyloxirane ${ }^{a}$

\begin{tabular}{|c|c|c|c|c|c|c|c|c|c|c|c|c|c|c|c|c|c|}
\hline \multicolumn{4}{|c|}{$6-31 \mathrm{G}$} & \multicolumn{4}{|c|}{$6-31 \mathrm{G}^{* *}$} & \multicolumn{4}{|c|}{ cc-pVDZ } & \multicolumn{4}{|c|}{ aug-cc-pVDZ } & \multicolumn{2}{|c|}{ experiment } \\
\hline$\omega^{\mathrm{HF}}$ & $\omega^{\mathrm{DFT}}$ & $\Delta_{z}^{\mathrm{HF}}$ & $\Delta_{z}^{\mathrm{DFT}}$ & $\omega^{\mathrm{HF}}$ & $\omega^{\mathrm{DFT}}$ & $\Delta_{z}^{\mathrm{HF}}$ & $\Delta_{z}^{\mathrm{DFT}}$ & $\omega^{\mathrm{HF}}$ & $\omega^{\mathrm{DFT}}$ & $\Delta_{z}^{\mathrm{HF}}$ & $\Delta_{z}^{\mathrm{DFT}}$ & $\omega^{\mathrm{HF}}$ & $\omega^{\mathrm{DFT}}$ & $\Delta_{z}^{\mathrm{HF}}$ & $\Delta_{z}^{\mathrm{DFT}}$ & $\omega$ & $\Delta_{z}$ \\
\hline 1676 & 1551 & 1.4 & -0.7 & 1687 & 1548 & 5.9 & 2.8 & 1665 & 1517 & 8.7 & 4.3 & 1659 & 1518 & 6.2 & 5.5 & 1498 & 1.0 \\
\hline 1652 & 1541 & -1.4 & -1.2 & 1625 & 1510 & -4.6 & -4.1 & 1595 & 1468 & -6.1 & -5.9 & 1595 & 1472 & -7.2 & -6.6 & 1460 & -2.7 \\
\hline 1641 & 1528 & 0.5 & 1.4 & 1611 & 1497 & 1.3 & 1.6 & 1580 & 1453 & 2.2 & 2.9 & 1578 & 1455 & 2.2 & 2.1 & 1450 & 0.8 \\
\hline 1592 & 1464 & 1.8 & 0.6 & 1586 & 1453 & 4.4 & 3.7 & 1563 & 1427 & 3.5 & 3.6 & 1561 & 1429 & 2.6 & 2.2 & 1403 & 3.1 \\
\hline 1567 & 1446 & 0.9 & 0.1 & 1535 & 1415 & -1.2 & -1.1 & 1507 & 1384 & -2.0 & -1.9 & 1504 & 1383 & -1.8 & -1.7 & 1365 & $b$ \\
\hline 1389 & 1288 & 0.0 & 0.0 & 1410 & 1300 & -0.3 & -0.5 & 1397 & 1284 & -0.6 & -0.7 & 1392 & 1284 & -1.0 & -0.9 & 1262 & $c$ \\
\hline 1318 & 1210 & 5.9 & 1.1 & 1316 & 1191 & 6.1 & 6.3 & 1296 & 1172 & 7.3 & 9.8 & 1291 & 1176 & 8.4 & 15.1 & 1164 & 4.0 \\
\hline 1296 & 1169 & -0.6 & -0.4 & 1296 & 1167 & 1.1 & -5.3 & 1276 & 1152 & 0.1 & -3.5 & 1274 & 1152 & 0.5 & -1.9 & 1140 & -3.3 \\
\hline 1276 & 1153 & -3.3 & -3.1 & 1271 & 1155 & -8.7 & 2.0 & 1259 & 1138 & -8.6 & 1.9 & 1258 & 1143 & -7.3 & -1.0 & 1135 & -3.8 \\
\hline 1218 & 1117 & -1.5 & -1.6 & 1240 & 1135 & -1.9 & -2.5 & 1223 & 1114 & -2.4 & -1.8 & 1217 & 1112 & -2.7 & 0.6 & 1101 & 1.2 \\
\hline 1139 & 1035 & 20.6 & 15.5 & 1141 & 1042 & 2.1 & -1.4 & 1128 & 1027 & 0.1 & -4.0 & 1123 & 1027 & -1.3 & -5.4 & 1020 & -5.1 \\
\hline 1049 & 969 & -5.6 & -4.3 & 1077 & 983 & -2.3 & -1.5 & 1071 & 974 & -2.1 & -0.7 & 1059 & 965 & -2.2 & -1.9 & 946 & 8.3 \\
\hline 995 & 909 & 2.8 & 3.3 & 990 & 909 & 2.6 & 2.4 & 979 & 898 & 2.0 & 2.1 & 973 & 896 & 2.6 & 3.2 & 892 & 4.6 \\
\hline 872 & 805 & 1.7 & 1.8 & 951 & 856 & 1.4 & 2.6 & 94 & 848 & 1.7 & 3. & 929 & 83 & 1.5 & 2.6 & 824 & 1.5 \\
\hline 806 & 713 & -0.6 & -1.4 & 858 & 782 & 0.2 & -1.5 & 852 & 774 & 0.2 & -1.8 & 844 & 762 & 0.1 & -2.0 & 742 & -3.1 \\
\hline 440 & 408 & 0.0 & 1.3 & 442 & 409 & -5.2 & -2.5 & 440 & 408 & -2.3 & -0.5 & 439 & 410 & 0.8 & 2.0 & 419 & $5.0^{a}$ \\
\hline 399 & 360 & 5.1 & 7.2 & 398 & 365 & 5.0 & 6.9 & 396 & 363 & 4.1 & 6.1 & 394 & 365 & 2.9 & 2.9 & 360 & $2.5^{d}$ \\
\hline 210 & 204 & 0.2 & -1.2 & 222 & 211 & 1.7 & 1.8 & 225 & 207 & 2.0 & 1.1 & 225 & 202 & 2.1 & -2.4 & 200 & $+^{b}$ \\
\hline
\end{tabular}

${ }^{a}$ The Hartree-Fock results have been taken from ref 7 and the experimental observations from ref 34 . The frequencies are reported in $\mathrm{cm}^{-1}$. ${ }^{b}$ Weak feature. ${ }^{c}$ Possible polarization artifact contribution. ${ }^{d}$ From ref 42.

and $\omega_{\mathrm{p}}$ is the harmonic frequency of this vibration. The derivatives of the generalized polarizability tensors with respect to displacements along the normal coordinate $Q_{\mathrm{p}}$ is evaluated at the equilibrium geometry $r_{\mathrm{e}}$.

An analytical approach to the calculation of first-order geometrical derivatives of frequency-dependent polarizabilities was recently presented at the Hartree-Fock level. ${ }^{27}$ However, because of the use of London-atomic orbitals in our calculations, this formalism cannot easily be extended to the electric dipolemagnetic dipole polarizability. We therefore calculate the geometrical derivatives of the polarizability tensors by numerical differentiation, as previously described. ${ }^{7}$

\section{Computational Details}

Two sets of calculations are presented in this paper. The first is a study of methyloxirane, where special attention is paid to basis-sets effects, as well as to the importance of electron correlation by comparison with previous Hartree-Fock results. ${ }^{7}$ Two types of basis sets are used: the $6-31 \mathrm{G}$ and $6-31 \mathrm{G}^{* *}$ basis sets by Pople and co-workers ${ }^{28-31}$ and the (augmented) correlation-consistent polarized valence double- $\zeta$ (aug)-cc-pVDZ basis sets of Dunning and Woon. ${ }^{32,33}$ For comparison, experimental data are included from ref 34.

The second study focuses on $\alpha$-pinene and trans-pinane. For these molecules, we compare both with recent experimental observations ${ }^{18}$ and with the SOS approach of Bour at the DFT level. ${ }^{17}$ Due to computational limitations, we have restricted ourselves to using the $6-31 \mathrm{G}$ and $6-31 \mathrm{G}^{* *}$ basis sets for these molecules.

In all calculations, we have used a step length of $0.001 a_{0}$ in the numerical differentiation, somewhat longer than we have used previously. ${ }^{7}$ However, test calculations indicated no change in the calculated CIDs. The numerical differentiation was performed by Cartesian displacements of all the atoms, without the use of translational and rotational invariance. The differentiated tensors were transformed to the normal coordinate basis using an analytically calculated Hessian. In all VROA calculations, the density was converged to a gradient of $10^{-7}$ and the iterative solution of the linear response equations to $5 \times 10^{-5}$. We used a dense grid consisting of 64 radial and 590 angular grid points for each atom. The B3LYP functional ${ }^{35}$ was used in all calculations, and all CIDs have been calculated for a wavelength of the incident light of $488.8 \mathrm{~nm}$.

For the calculation of the CIDs, we have used a local version of the Dalton program, ${ }^{24}$ using the recent implementation of linear response functions for the calculation of optical rotation at the DFT level. ${ }^{19}$ However, Dalton is currently incapable of calculating analytical Hessians at the DFT level, for which we instead used Gaussian98. ${ }^{36}$ It is here important to note that the B3LYP functional as defined in Dalton differs from that used in Gaussian98. Whereas the latter uses the functional form III of the Vosko, Wilk, and Nusair (VWN) correlation functional, ${ }^{37}$ Dalton uses functional form $\mathrm{V}$ of the same paper. However, test calculations of optical rotations show only minor differences compared to the work of Stephens et al., ${ }^{38}$ and we will assume that this agreement also holds for the vibrational frequencies. The Hessians were transformed to Dalton format using an interface routine written by Hangartner, ${ }^{39}$ which is distributed with the Dalton program.

\section{Results}

4.1. Methyloxirane. Our results for $R$-methyloxirane are collected in Table 1, where we also report the Hartree-Fock results of ref 7 and the experimental results of ref 34 . Some of the Hartree-Fock results in Table 1 have been obtained by us to investigate more carefully the importance of diffuse functions on the VROA calculation.

The calculated harmonic frequencies listed in Table 1 confirm the established knowledge that DFT and, in particular, the B3LYP functional, gives better agreement with experiment than Hartree-Fock does for the vibrational frequencies. ${ }^{13}$ There are some changes in the frequencies from $6-31 \mathrm{G}$ to $6-31 \mathrm{G}^{* *}$, but overall the cc-pVDZ basis set provides a significantly better agreement with experiment than do the Pople basis sets. However, as we compare experimental fundamental frequencies with theoretical harmonic frequencies (and ignore possible solvent effects), exact agreement cannot be expected. The differences between the cc-pVDZ and aug-cc-pVDZ frequencies are negligible. This suggests that augmentation has little influence on the vibrational frequencies.

Turning our attention to the calculated VROA CIDs for rightangle scattering, we note that Hartree-Fock and DFT are in 
much better agreement than was the case for the frequencies. Although there are still substantial differences, most of the qualitative intensity features of the spectrum are reproduced by both Hartree-Fock theory and DFT. This observation suggests that the most important aspect for the prediction of a VROA spectrum is an accurate force field and that it should be possible to use the DFT/B3LYP force field together with Hartree-Fock intensities, as done for instance in ref 15 . We shall return to this point shortly.

Despite the similarities between the HF and DFT intensities, there are some important differences. In particular, the HF and DFT intensities differ in sign for several of the observed transitions. For the larger basis sets, these differences are mostly confined to low-intensity vibrational modes, thus possibly arising from a partial cancellation of the three contributing terms to the VROA intensity. Still, the differences between HF and DFT are to some extent less severe than the errors incurred by using small basis sets.

Whereas small differences are observed between 6-31G** and cc-pVDZ for the frequencies, larger differences are found for the intensities. It is discouraging to note that there are also significant changes in the intensities from cc-pVDZ to aug-ccpVDZ. In particular, at the DFT/aug-cc-pVDZ level the number of modes calculated with incorrect signs decreases to two (those at 965 and $202 \mathrm{~cm}^{-1}$ ), whereas for most other models at least four differences in sign can be observed. Fortuitously, only three bands differ in sign at the HF/6-31G level.

In the first theoretical investigation of $R$-methyloxirane, Bose et al. suggested interchanging the bands at 1218 and $1139 \mathrm{~cm}^{-1}$, as these bands at the HF/6-31G level were predicted to be very strong with the opposite sign of experiment. However, the intensity of these bands were reduced significantly in the 6-31G* basis, ${ }^{34}$ indicating that basis-set effects may be substantial. Indeed, this has been corroborated by the use of the even larger aug-cc-pVDZ basis in ref 7, where both bands become negative. The results presented in Table 1 for these bands suggest that proper treatment of electron correlation (best represented at the B3LYP/aug-cc-pVDZ level) is necessary for a reliable computation of the signs and relative intensities.

Although the cost of the B3LYP calculation scales similarly as for the HF calculation, the DFT method involves a numerical quadrature in addition to the Coulomb and exchange integrals, thus making the calculation always more expensive. The cost is further increased by the need of a fine integration grid to avoid problems with inaccuracies in the numerical differentiation. It is therefore of practical interest to explore the possibility of combining the DFT force field with the HF polarizability derivatives, as in Table 2, where we report the results obtained by evaluating the HF ROA intensities both at the HF and B3LYP minimum geometry, with the aug-cc-pVDZ basis. Although pure HF and B3LYP computations differ significantly (see Table 1), the combination of the B3LYP force field and the HF CIDs provides results of the same quality as those obtained with pure DFT. Moreover, there are no significant differences between CIDs evaluated at the HF and B3LYP minima. Because, however, the force field needs to be determined at the B3LYP minimum, the DFT geometry appears more convenient.

There are two minor exceptions to the agreement between DFT and the mixed DFT/HF scheme. For the frequency at 1135 $\mathrm{cm}^{-1}$, use of the Hartree-Fock geometry in the pure DFT scheme produces an intensity with a sign different from that of the other methods and that of experiment. The situation is different for the mode at $200 \mathrm{~cm}^{-1}$, where only the intensities
TABLE 2: Calculated ROA Intensities for $\boldsymbol{R}$-Methyloxirane ${ }^{a}$

\begin{tabular}{rrrrrrr}
\hline & \multicolumn{2}{c}{ HF geometry } & & \multicolumn{2}{c}{ DFT geometry } & \\
\cline { 2 - 3 }$\omega_{\exp }$ & \multicolumn{1}{c}{$\Delta_{z}^{\mathrm{HF}}$} & $\Delta_{z}^{\mathrm{DFT}}$ & & $\Delta_{z}^{\mathrm{HF}}$ & $\Delta_{z}^{\mathrm{DFT}}$ & $\Delta_{z}^{\exp }$ \\
\hline 1498 & 6.2 & 6.1 & 6.2 & 5.5 & 1.0 \\
1460 & -7.2 & -6.3 & -6.6 & -6.6 & -2.7 \\
1450 & 2.2 & 2.1 & 2.0 & 2.1 & 0.8 \\
1403 & 2.6 & 2.5 & 2.5 & 2.2 & 3.1 \\
1365 & -1.8 & -2.5 & -2.1 & -1.7 & $b$ \\
1262 & -1.0 & -1.0 & -1.0 & -0.9 & $c$ \\
1164 & 8.4 & 17.0 & 16.6 & 15.1 & 4.0 \\
1140 & 0.5 & -1.8 & -1.8 & -1.9 & -3.3 \\
1135 & -7.3 & 0.2 & -0.3 & -1.0 & -3.8 \\
1101 & -2.7 & -2.2 & -1.6 & 0.6 & 1.2 \\
1020 & -1.3 & -3.6 & -4.2 & -5.4 & -5.1 \\
946 & -2.2 & -2.8 & -2.9 & -1.9 & 8.3 \\
892 & 2.6 & 3.2 & 3.0 & 3.2 & 4.6 \\
824 & 1.5 & 1.9 & 1.9 & 2.6 & 1.5 \\
742 & 0.1 & -0.6 & -0.6 & -2.0 & -3.1 \\
419 & 0.8 & 1.0 & 1.1 & 2.0 & $5.0^{d}$ \\
360 & 2.9 & 3.1 & 3.2 & 2.9 & $2.5^{d}$ \\
200 & 2.1 & -1.9 & 1.2 & -2.4 & $+^{b}$
\end{tabular}

${ }^{a}$ The Hartree-Fock results have been taken from ref 7 and the experimental observations from ref 34 . The frequencies are reported in $\mathrm{cm}^{-1}$. In all cases a DFT/B3LYP force field has been used. The superscript in $\Delta_{z}^{\mathrm{wf}}$ indicates the wave function used for calculating the VROA intensities. ${ }^{b}$ Weak feature. ${ }^{c}$ Possible polarization artifact contribution. ${ }^{d}$ From ref 42 .

calculated in the mixed approach has the same sign as that observed in experiment. Because of the flat potential for this mode, which involves large relative atomic displacements as it is dominated by methyl rotation, small differences in the equilibrium geometry may give large differences in the VROA intensities. We therefore cannot draw definite conclusions for this vibrational mode. Also, because the internal rotation is almost free, the perturbational treatment used here for calculating the VROA intensities cannot be expected to be accurate.

4.2. $\alpha$-Pinene and trans-Pinane. Our calculated B3LYP frequencies and VROA circular intensity differences $I_{180}^{\mathrm{R}}-$ $I_{180}^{\mathrm{L}}$ for $(1 S)-(-)-\alpha$-pinene in the $6-31 \mathrm{G}$ and $6-31 \mathrm{G}^{* *}$ basis sets are collected in Table 3, along with the results of the sumover-states approach and the mixed HF/DFT scheme of Bour. ${ }^{17}$ The force field and geometry of all calculations in ref 17 were obtained using the BPW91 functional and a TZ2P basis; their results are therefore not directly comparable with ours, obtained with the B3LYP functional and the 6-31G and 6-31G** basis sets. However, the SOS CIDs of ref 17 were obtained at the B3LYP/6-31G** level. The mixed HF/DFT calculations employed Hartree-Fock intensities using the 6-31G** basis.

There are significant differences in the frequencies at the BPW91/TZ2P and B3LYP/6-31G** levels, whereas the B3LYP frequencies are rather similar for the $6-31 \mathrm{G}$ and the $6-31 \mathrm{G}^{* *}$ basis sets. In view of the discussion in the previous section, the main reason for the differences is probably the use of a much larger basis for the calculation of the molecular force field in refs 17 and 40.

Considering the differences in the force field, the SOS scheme performs surpringly well compared to our full DFT calculation. The main features of the spectrum are in good agreement with the B3LYP/6-31G** intensities, although the magnitude of the CIDs are often overestimated. For a few bands, the different models predict different signs of the CIDs, in particular in the $1100-900 \mathrm{~cm}^{-1}$ region. For most bands in this region, the HF/ DFT results of ref 17 and our B3LYP/6-31G results agree with the "reference" B3LYP/6-31G** results, indicating that the SOS+Polar scheme does not reproduce the CIDs correctly in this region. 
TABLE 3: Vibrational Frequencies $\left(\right.$ in $\mathrm{cm}^{-1}$ ) and Backscattering CIDs of $\alpha$-Pinene

\begin{tabular}{|c|c|c|c|c|c|c|}
\hline \multicolumn{3}{|c|}{ vibrational frequency } & \multicolumn{4}{|c|}{ CIDs } \\
\hline ref 17 & $\begin{array}{l}\text { B3LYP/ } \\
6-31 \mathrm{G}\end{array}$ & $\begin{array}{l}\text { B3LYP/ } \\
6-31 G^{*} *\end{array}$ & $\begin{array}{c}\text { Polar+ } \\
\text { SOS }^{17}\end{array}$ & $\begin{array}{c}\text { mixed } \\
\text { HF/DFT }^{17}\end{array}$ & $\begin{array}{c}\text { B3LYP/ } \\
6-31 G\end{array}$ & $\begin{array}{l}\text { B3LYP/ } \\
6-31 \mathrm{G}^{* *}\end{array}$ \\
\hline 1663 & 1734 & 1732 & -34 & -12 & -8 & -9 \\
\hline 1477 & 1561 & 1531 & 2 & 11 & 16 & 8 \\
\hline 1468 & 1555 & 1526 & -62 & -71 & -34 & -25 \\
\hline 1455 & 1541 & 1511 & -19 & -32 & -48 & -44 \\
\hline 1451 & 1538 & 1505 & -195 & -257 & -56 & -103 \\
\hline 1448 & 1534 & 1502 & 307 & 413 & 281 & 256 \\
\hline 1440 & 1527 & 1496 & 77 & 100 & 32 & 45 \\
\hline 1437 & 1524 & 1490 & 4 & 8 & 24 & 21 \\
\hline 1432 & 1524 & 1490 & -174 & -255 & -276 & -217 \\
\hline 1373 & 1463 & 1431 & 3 & 9 & 8 & 7 \\
\hline 1365 & 1460 & 1426 & 9 & 18 & 7 & 13 \\
\hline 1354 & 1445 & 1413 & 5 & 1 & 6 & 4 \\
\hline 1326 & 1394 & 1375 & -3 & 0 & 5 & 5 \\
\hline 1312 & 1382 & 1366 & -4 & 6 & 9 & 15 \\
\hline 1292 & 1358 & 1345 & -5 & -8 & 5 & -6 \\
\hline 1250 & 1311 & 1305 & 13 & 10 & -12 & 1 \\
\hline 1235 & 1304 & 1283 & 8 & 11 & 4 & -1 \\
\hline 1206 & 1274 & 1258 & 34 & 37 & 25 & 17 \\
\hline 1199 & 1257 & 1244 & -9 & -32 & -4 & -13 \\
\hline 1190 & 1250 & 1237 & -44 & -39 & -30 & -19 \\
\hline 1169 & 1238 & 1215 & 2 & 0 & 5 & -1 \\
\hline 1155 & 1211 & 1195 & 19 & 25 & 14 & 19 \\
\hline 1114 & 1170 & 1154 & -17 & -8 & -16 & -8 \\
\hline 1087 & 1146 & 1134 & 21 & 32 & -1 & -5 \\
\hline 1074 & 1127 & 1113 & -7 & 43 & 49 & 37 \\
\hline 1051 & 1112 & 1085 & 15 & 2 & 7 & -1 \\
\hline 1031 & 1097 & 1068 & -40 & -30 & -26 & -14 \\
\hline 1026 & 1074 & 1061 & 18 & -15 & -12 & -2 \\
\hline 1003 & 1059 & 1039 & -12 & -5 & -10 & 0 \\
\hline 989 & 1047 & 1024 & -1 & 8 & -5 & -5 \\
\hline 944 & 997 & 975 & -30 & -32 & 12 & -12 \\
\hline 939 & 988 & 975 & -29 & 12 & 4 & 16 \\
\hline 923 & 971 & 954 & 6 & -2 & -9 & -3 \\
\hline 916 & 953 & 944 & 47 & 60 & 28 & 29 \\
\hline 893 & 928 & 923 & 33 & 35 & 26 & 18 \\
\hline 873 & 905 & 901 & -8 & -16 & -23 & -19 \\
\hline 830 & 854 & 856 & -15 & -25 & -11 & -8 \\
\hline 800 & 832 & 829 & 5 & 10 & -13 & 1 \\
\hline 777 & 824 & 813 & -65 & -95 & -34 & -44 \\
\hline 758 & 786 & 783 & 24 & 45 & 21 & 20 \\
\hline 655 & 680 & 674 & 5 & 17 & 6 & 7 \\
\hline 605 & 636 & 626 & 83 & 60 & 31 & 25 \\
\hline 556 & 581 & 574 & -55 & -48 & -22 & -17 \\
\hline 473 & 495 & 486 & 3 & 11 & 7 & 7 \\
\hline 451 & 473 & 465 & 18 & -5 & -1 & -1 \\
\hline 410 & 433 & 429 & -8 & -3 & -3 & -4 \\
\hline
\end{tabular}

In Figure 1, we have plotted the experimental spectrum of $(1 S)-(-)$ - $\alpha$-pinene from ref 18 together with a simulated spectrum based on our $\mathrm{B} 3 \mathrm{LYP} / 6-31 \mathrm{G}^{* *}$ results, using a Lorentzian line width of $8 \mathrm{~cm}^{-1}$. We note that the experimental spectrum has a rather low signal-to-noise $(\mathrm{S} / \mathrm{N})$ ratio, and that VROA spectra of this molecule, as well as trans-pinane, with a much improved $\mathrm{S} / \mathrm{N}$ ratio have been published by Vargek, Freedman, and Nafie. ${ }^{41}$ However, despite the low S/N ratio, it can easily be seen that the simulated spectrum is in good agreement with the experimental spectrum we have plotted in Figure 1.

In Figure 2, we have plotted the experimental Raman spectrum of $(1 S)-(-)-\alpha$-pinene together with our simulated spectrum obtained using B3LYP/6-31G**. For the simulated Raman spectra, we have plotted the absolute differential Raman scattering cross section, ${ }^{9}$ setting the height of the peak at 1450 $\mathrm{cm}^{-1}$ to approximately the same height as in the experimental Raman spectrum. Although many of the qualitative features of the Raman spectrum are correctly reproduced, we note that there
TABLE 4: Vibrational Frequencies $\left(\right.$ in $\mathrm{cm}^{-1}$ ) and Backscattering CIDs of trans-Pinane

\begin{tabular}{|c|c|c|c|c|}
\hline \multicolumn{2}{|c|}{ vibrational frequency } & \multicolumn{3}{|c|}{ CIDs } \\
\hline B3LYP/ & B3LYP/ & Polar+ & B3LYP/ & B3LYP/ \\
\hline $6-31 G$ & $6-31 G^{* *}$ & $\operatorname{SOS}^{17}$ & $6-31 \mathrm{G}$ & $6-31 G^{* *}$ \\
\hline 1568 & 1537 & -41 & -58 & -50 \\
\hline 1561 & 1530 & 22 & 51 & 24 \\
\hline 1549 & 1518 & 84 & 68 & 55 \\
\hline 1548 & 1517 & -39 & -23 & -3 \\
\hline 1542 & 1512 & 47 & -50 & 42 \\
\hline 1542 & 1510 & -53 & 39 & -47 \\
\hline 1538 & 1504 & -22 & -5 & 11 \\
\hline 1532 & 1498 & -67 & 3 & -76 \\
\hline 1529 & 1497 & 46 & -44 & 25 \\
\hline 1464 & 1433 & 6 & 4 & 3 \\
\hline 1454 & 1422 & 2 & -4 & -1 \\
\hline 1446 & 1415 & 1 & 2 & 4 \\
\hline 1412 & 1397 & 10 & 47 & 18 \\
\hline 1401 & 1379 & -7 & -41 & 3 \\
\hline 1383 & 1362 & -36 & -5 & -29 \\
\hline 1377 & 1351 & 14 & 22 & 21 \\
\hline 1346 & 1337 & 66 & 55 & 50 \\
\hline 1340 & 1324 & -107 & -83 & -92 \\
\hline 1316 & 1304 & -7 & -11 & 13 \\
\hline 1290 & 1273 & 14 & 22 & 14 \\
\hline 1271 & 1254 & -3 & -14 & -12 \\
\hline 1258 & 1242 & 8 & 15 & 4 \\
\hline 1253 & 1235 & -13 & -4 & -12 \\
\hline 1241 & 1229 & -6 & -15 & 1 \\
\hline 1211 & 1195 & -25 & -5 & -49 \\
\hline 1197 & 1182 & -1 & 5 & 7 \\
\hline 1147 & 1127 & 57 & 60 & 57 \\
\hline 1128 & 1112 & 26 & 33 & 19 \\
\hline 1122 & 1102 & -25 & -41 & -22 \\
\hline 1088 & 1076 & -18 & -27 & -16 \\
\hline 1070 & 1057 & 6 & -19 & -15 \\
\hline 1048 & 1027 & 28 & 19 & 24 \\
\hline 1029 & 1014 & -27 & 14 & 6 \\
\hline 1026 & 1012 & 25 & 23 & 8 \\
\hline 988 & 969 & -76 & -21 & -62 \\
\hline 981 & 965 & 19 & -4 & 31 \\
\hline 968 & 952 & -4 & -8 & -4 \\
\hline 937 & 933 & 95 & 56 & 61 \\
\hline 916 & 907 & -18 & -6 & -11 \\
\hline 895 & 890 & 67 & 34 & 43 \\
\hline 863 & 864 & -141 & -105 & -93 \\
\hline 832 & 830 & 2 & -3 & 0 \\
\hline 799 & 789 & 32 & 47 & 39 \\
\hline 790 & 785 & -18 & -1 & -6 \\
\hline 668 & 664 & 0 & 1 & -5 \\
\hline 586 & 576 & 1 & -4 & -4 \\
\hline 533 & 522 & 5 & 9 & 7 \\
\hline 487 & 480 & -7 & -6 & -6 \\
\hline 460 & 452 & 1 & 1 & 1 \\
\hline 436 & 431 & 0 & 0 & 0 \\
\hline
\end{tabular}

are significant discrepancies between the experimental Raman spectrum and our simulated spectrum, in particular in the regions 1400-1200 and 950-850 $\mathrm{cm}^{-1}$. This might indicate a need for more diffuse functions to properly describe the polarizability derivatives but may also be due to solvent effects (not included in our study) or conformational flexibility of the molecule. Considering these problems for the Raman spectrum, the agreement observed for the VROA spectrum using the $6-31 \mathrm{G}^{* *}$ basis set is promising.

In Table 4, we have collected our results for the frequencies and CIDs in (-)-trans-pinane. For this molecule, we have also calculated the CIDs using the SOS+Polar model of ref 17 at the B3LYP/6-31G** level, enabling a direct comparison of the SOS+Polar results with the full DFT results.

In view of its low computational cost, the SOS+Polar model performs very well. Interestingly, for many of the CIDs where 


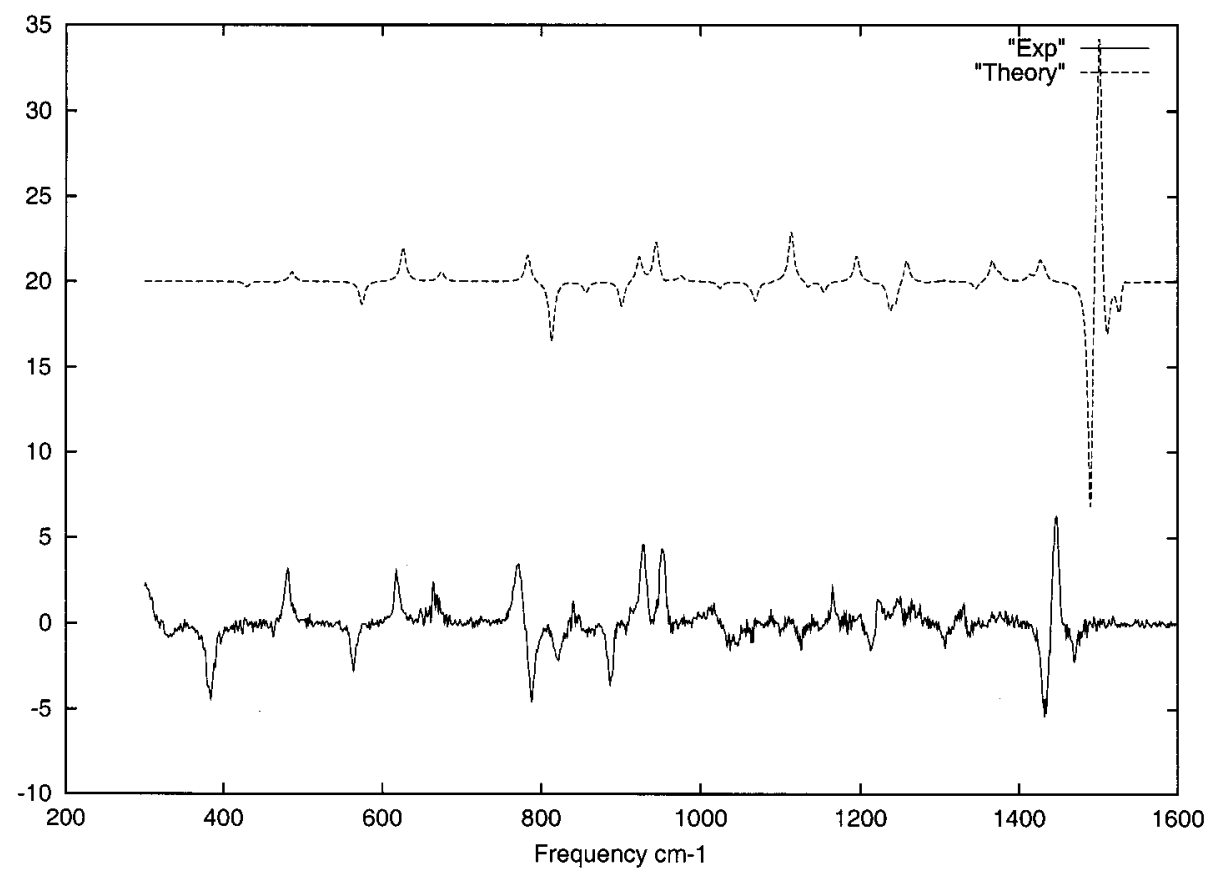

Figure 1. Experimental (from ref 18) and simulated spectra of VROA backscattering of $\alpha$-pinene. Units on the $y$ axis are arbitrary. A line width of $8 \mathrm{~cm}^{-1}$ was used in the simulation of the theoretical spectrum.

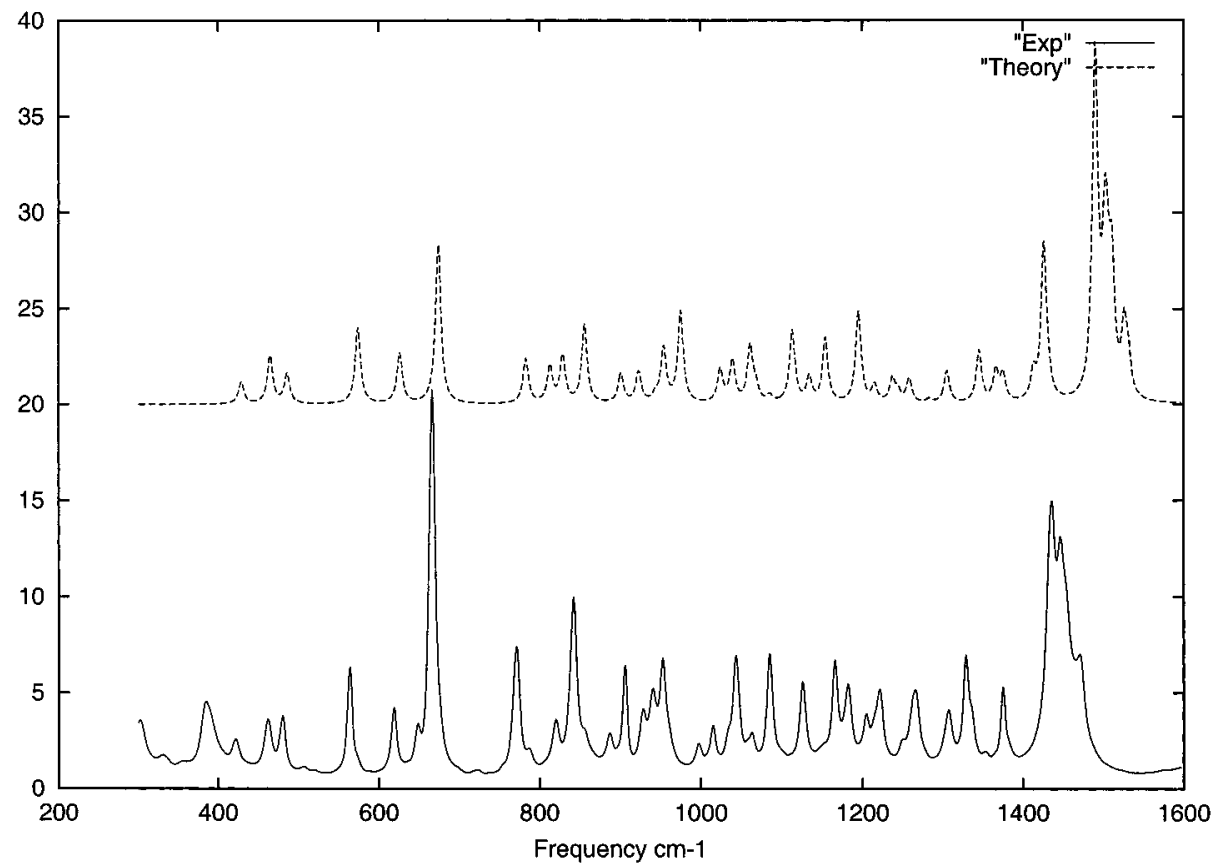

Figure 2. Experimental (from ref 18) and simulated Raman spectra of $\alpha$-pinene. Units on the $y$ axis are arbitrary. A line width of $8 \mathrm{~cm}^{-1}$ was used in the simulation of the theoretical spectrum.

the SOS+Polar model differs from the DFT/6-31G** approach, it agrees more with B3LYP/6-31G. In some sense, therefore, the SOS model mimicks the effect of using smaller basis sets, presumable leading to a poorer description of the excited states. In general, the SOS+Polar model works better for vibrational modes with large intensity differences, where it often gives better agreement with the full $\mathrm{B} 3 \mathrm{LYP} / 6-31 \mathrm{G} * *$ results than with the B3LYP/6-31G results.

In Figure 3, we have plotted a simulation of our B3LYP/6$31 \mathrm{G}^{* *}$ and SOS+Polar results together with the experimental VROA spectrum of ref 18. As for $\alpha$-pinene, the experimental spectrum is well reproduced by the $\mathrm{B} 3 \mathrm{LYP} / 6-31 \mathrm{G}^{* *}$ calculation, and also by the SOS+Polar model. The SOS+Polar approach thus appears to be a fast and inexpensive tool for quickly providing theoretical CIDs that may help in the first assignment of the experimental VROA spectra. On the other hand, the consistent linear response DFT ROA computation is clearly necessary to reproduce most of the finer spectral features. These include the negative signal around $650 \mathrm{~cm}^{-1}$ missing for the SOS model, as well as the small positive bands around 940 and $1140 \mathrm{~cm}^{-1}$, or the positive signal around $1330 \mathrm{~cm}^{-1}$, overestimated by SOS.

In Figure 4 we have plotted the experimental and theoretical Raman spectrum of (-)-trans-pinane. The simulated Raman spectrum for this molecule seems to be in better agreement with the general intensity features of the experimental spectrum than 


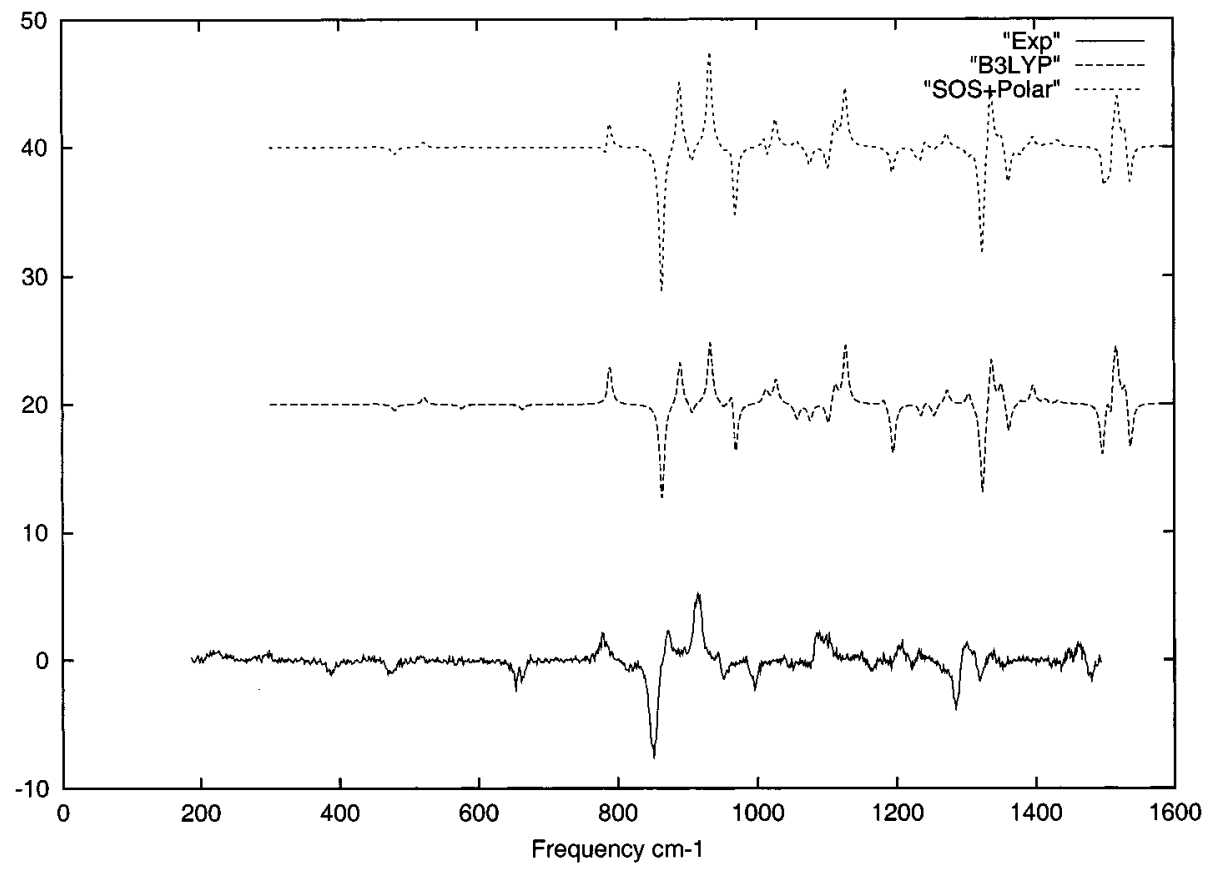

Figure 3. Experimental (from ref 18) and simulated spectra of VROA backscattering of trans-pinane. Units on the $y$ axis are arbitrary. A line width of $8 \mathrm{~cm}^{-1}$ was used in the simulation of the theoretical spectrum.

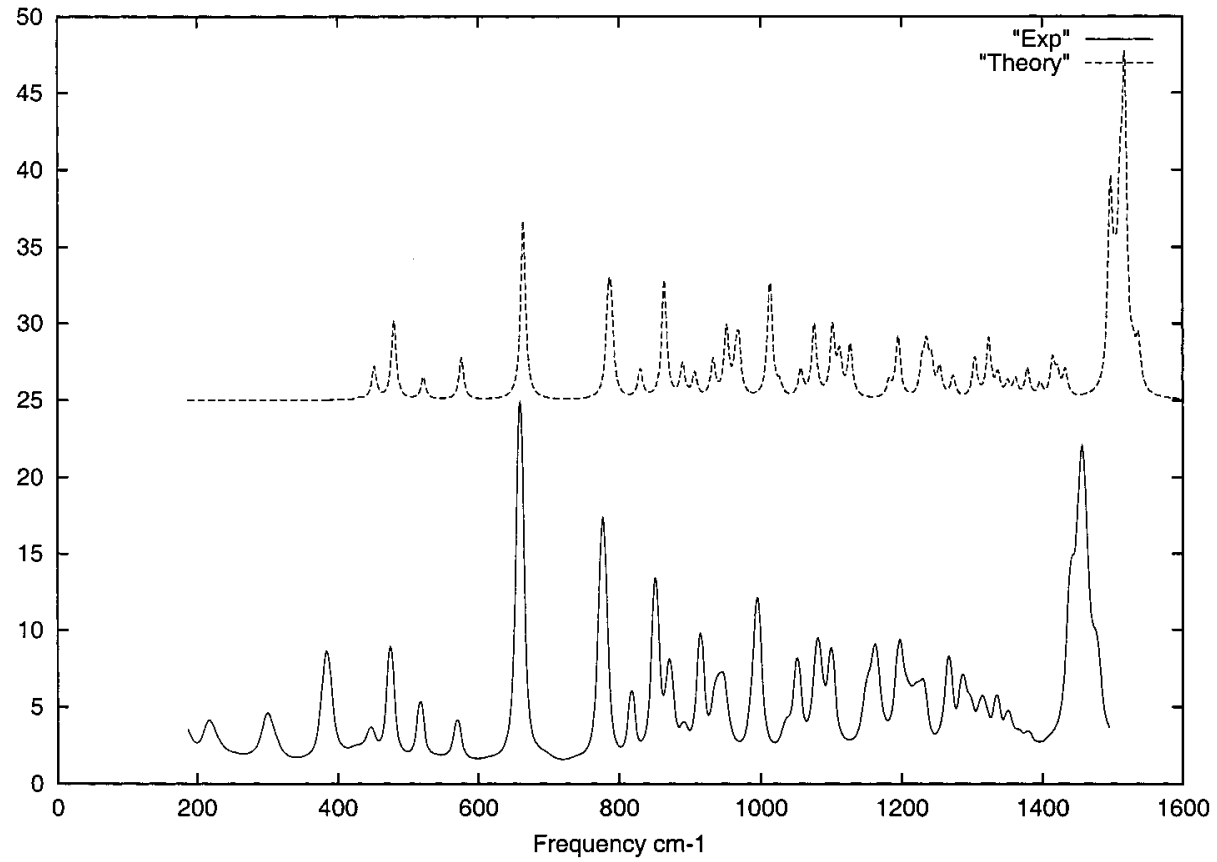

Figure 4. Experimental (from ref 18) and simulated Raman spectra of trans-pinane. Units on the $y$ axis are arbitrary. A line width of $8 \mathrm{~cm}^{-1}$ was used in the simulation of the theoretical spectrum.

was the case for $(1 S)-(-)-\alpha$-pinene. However, in the region $1400-1200 \mathrm{~cm}^{-1}$, the agreement with experiment is rather poor.

\section{Concluding Remarks}

We have presented the first gauge-origin independent calculations of vibrational Raman optical activity consistently using linear response methods implemented within density-functional theory; the frequency dependence has been included in the calculation, and gauge-origin independence has been ensured by the use of London atomic orbitals. It has been confirmed that use of the DFT/B3LYP force field instead of a HartreeFock force field leads to a significant improvment in the harmonic vibrational frequencies. Although the use of DFT also improves the VROA intensities, the differences between HF and DFT for the CIDs are in general much smaller than for the frequencies. However, to reproduce the finer details of the VROA spectrum, the presented DFT linear response approach appear to be the most accurate method currently available.

Acknowledgment. This work has been supported by the Norwegian Research Council through a grant of computer time from the Program for Supercomputing. P.B. acknowledges support from the Grant Agency of the Czech Republic (203/ 01/0031). We are indebted to Prof. V. Baumruk for providing 
us with the raw data for the experimental VROA spectra for $\alpha$-pinene and trans-pinane.

\section{References and Notes}

(1) Bell, A. F.; Hecht, L.; Barron, L. D. J. Am. Chem. Soc. 1998, 120,5820 .

(2) Barron, L. D.; Hecht, L.; Bell, A. F. Appl. Spectrosc. 1996, 50, 619.

(3) Barron, L. D.; Hecht, L.; Blanch, E. W.; Bell, A. F. Prog. Biophys. Mol. Bio. 2000, 73, 1 .

(4) See the company homepage at http://www.btools.com.

(5) Nafie, L. A. Аппи. Rev. Phys. Chem. 1997, 48, 357.

(6) Olsen, J.; Jørgensen, P. J. Chem. Phys. 1985, 82, 3235.

(7) Helgaker, T.; Ruud, K.; Bak, K. L.; Jørgensen, P.; Olsen, J. Faraday Discuss. 1994, 99, 165

(8) Amos, R. D. Chem. Phys. Lett. 1982, 87, 23.

(9) Polavarapu, P. L. J. Phys. Chem. 1990, 94, 8106.

(10) London, F. J. Phys. Radium 1937, 8, 397.

(11) Ditchfield, R. J. Chem. Phys. 1972, 56, 5688.

(12) Wolinski, K.; Hinton, J. F.; Pulay, P. J. Am. Chem. Soc. 1990 112,8251 .

(13) Kim, K.; Jordan, K. D. J. Phys. Chem. 1994, 98, 10089.

(14) Stanton, J. F.; Gauss, J. Int. Rev. Phys. Chem. 2000, 19, 61.

(15) Jalkanen, K. J.; Nieminen, R. M.; Frimand, K.; Bohr, J.; Bohr, H.; Wade, R. C.; Tajkhorshid, E.; Suhai, S. Chem. Phys. 2001, 265, 125.

(16) Bouř, P. Chem. Phys. Lett. 1998, 288, 363

(17) Bouř, P. J. Comput. Chem. 2001, 22, 426.

(18) Bouř, P.; Baumruk, V.; Hanzliková, J. Collect. Czech. Chem Commun 1997, 62, 1384

(19) Ruud, K.; Helgaker, T. Chem. Phys. Lett. 2002, 352, 533.

(20) Barron, L. D.; Buckingham, A. D. Mol. Phys. 1971, 20, 1111

(21) Atkins, P. W.; Barron, L. D. Mol. Phys. 1969, 16, 453.

(22) Olsen, J.; Bak, K. L.; Ruud, K.; Helgaker, T.; Jørgensen, P. Theor. Chim. Acta 1995, 90, 421.

(23) Polavarapu, P. L. Mol. Phys. 1997, 91, 551.

(24) Helgaker, T.; Jensen, H. J. Aa.; Jørgensen, P.; Olsen, J.; Ruud, K.; Agren, H.; Auer, A. A.; Bak, K. L.; Bakken, V.; Christiansen, O.; Coriani, S.; Dahle, P.; Dalskov, E. K.; Enevoldsen, T.; Fernandez, B.; Hättig, C.; Hald, K.; Halkier, A.; Heiberg, H.; Hettema, H.; Jonsson, D.; Kirpekar, S.; Kobayashi, R.; Koch, H.; Mikkelsen, K. V.; Norman, P.; Packer, M. J.; Pedersen, T. B.; Ruden, T. A.; Sanchez, A.; Saue, T.; Sauer, S. P. A.;
Schimmelpfennig, B.; Sylvester-Hvid, K. O.; Taylor, P. R.; Vahtras, O. Dalton, an ab initio electronic structure program, release 1.2. See http:// www.kjemi.uio.no/software/dalton/dalton.html, 2001.

(25) Barron, L. D. Molecular light scattering and optical activity; Cambridge University Press: Cambridge, 1982.

(26) Placzek, G. Handbuch der Radiologie 234, 6, 205

(27) Quinet, O.; Champagne, B. J. Chem. Phys. 2001, 115, 6293.

(28) Hehre, W. J.; Ditchfield, R.; Pople, J. A. J. Chem. Phys. 1972 $56,2257$.

(29) Hariharan, P. C.; Pople, J. A. Theor. Chim. Acta 1973, 28, 213.

(30) Gordon, M. S. Chem. Phys. Lett. 1980, 76, 163.

(31) Clark, T.; Chandrasekhar, J.; Spitznagel, G. W.; Schleyer, P. v. R. J. Comput. Chem. 1983, 4, 294.

(32) Dunning, T. H., Jr. J. Chem. Phys. 1989, 90, 1007.

(33) Woon, D. E.; Dunning, T. H., Jr. J. Chem. Phys. 1994, 100, 2975

(34) Bose, P. K.; Polavarapu, P. L.; Barron, L. D.; Hecht, L. J. Phys. Chem. 1990, 94, 1734.

(35) Stephens, P. J.; Devlin, F. J.; Chabalowski, C. F.; Frisch, M. J. J. Phys. Chem. 1994, 98, 11623.

(36) Frisch, M. J.; Trucks, G. W.; Schlegel, H. B.; Scuseria, G. E.; Robb, M. A.; Cheeseman, J. R.; Zakrzewski, V. G.; Montgomery, J. A.; Jr., Stratmann, R. E.; Burant, J. C.; Dapprich, S.; Millam, J. M.; Daniels, A. D.; Kudin, K. N.; Strain, M. C.; Farkas, O.; Tomasi, J.; Barone, V.; Cossi, M.; Cammi, R.; Mennucci, B.; Pomelli, C.; Adamo, C.; Clifford, S.; Ochterski, J.; Petersson, G. A.; Ayala, P. Y.; Cui, Q.; Morokuma, K.; Malick, D. K.; Rabuck, A. D.; Raghavachari, K.; Foresman, J. B.; Cioslowski, J.; Ortiz, J. V.; Stefanov, B. B.; Liu, G.; Liashenko, A.; Piskorz, P.; Komaromi, I.; Gomperts, R.; Martin, R. L.; Fox, D. J.; Keith, T.; Al-Laham, M. A.; Peng, C. Y.; Nanayakkara, A.; Gonzalez, C.; Challacombe, M.; Gill, P. M. W.; Johnson, B.; Chen, W.; Wong, M. W.; Andres, J. L.; Gonzalez, C.; Head-Gordon, M.; Replogle, E. S.; Pople, J. A. Gaussian 98, revision A.5; Gaussian, Inc.: Pittsburgh, PA, 1998.

(37) Vosko, S. J.; Wilk, L.; Nusair, M. Can. J. Phys 1980, 58, 1200.

(38) Stephens, P. J.; Devlin, F. J.; Cheeseman, J. R.; Frisch, M. J. J. Phys. Chem. A 2001, 105, 5356.

(39) Hangartner, G. FChk2Hes, unpublished, 1997.

(40) Devlin, F. J.; Stephens, P. J.; Cheeseman, J. R.; Frisch, M. J. J. Phys. Chem. A 1997, 101, 6322.

(41) Vargek, M.; Freedman, T. B.; Nafie, L. A. J. Raman Spectrosc. 1997, 28, 627.

(42) Barron, L. D.; Vrbancich, J. Mol. Phys. 1983, 48, 833 